\title{
COMPLETE MAXIMAL SPACELIKE SURFACES IN AN ANTI-DE SITTER SPACE $\mathbf{H}_{2}^{4}(c)^{*}$ \\ QING-MING CHENG
}

Department of Mathematics, Faculty of Science, Josai University, Sakado, Saitama 350-0295, Japan

e-mail: cheng@math.josai.ac.jp

(Received 14 August, 1998)

\begin{abstract}
In this paper, we prove that if $M^{2}$ is a complete maximal spacelike surface of an anti-de Sitter space $\mathbf{H}_{2}^{4}(c)$ with constant scalar curvature, then $S=0$, $S=\frac{-10 c}{11}, S=\frac{-4 c}{3}$ or $S=-2 c$, where $S$ is the squared norm of the second fundamental form of $M^{2}$. Also

(1) $S=0$ if and only if $M^{2}$ is the totally geodesic surface $\mathbf{H}^{2}(c)$;

(2) $S=\frac{-4 c}{3}$ if and only if $M^{2}$ is the hyperbolic Veronese surface;

(3) $S=-2 c$ if and only if $M^{2}$ is the hyperbolic cylinder of the totally geodesic surface $\mathbf{H}_{1}^{3}(c)$ of $\mathbf{H}_{2}^{4}(c)$.

1991 Mathematics Subject Classification. 53C40, 53C42
\end{abstract}

1. Introduction. Let $M_{p}^{n+p}(c)$ be an $(n+p)$-dimensional connected semiRiemannian manifold of index $p$ and of constant curvature $c$, which is called as an indefinite space form of index $p$. The standard models of indefinite space forms are given as follows. In an $(n+p)$-dimensional real vector space $\mathbf{R}^{n+p}$ with the standard basis, the scalar product $\langle$,$\rangle is given by$

$$
\langle x, y\rangle=\sum_{i=1}^{n} x_{i} y_{i}-\sum_{j=n+1}^{n+p} x_{j} y_{j},
$$

where $x=\left(x_{1}, x_{2}, \cdots, x_{n+p}\right)$ and $y=\left(y_{1}, y_{2}, \cdots, y_{n+p}\right)$. Then $\left(\mathbf{R}^{n+p},\langle\rangle,\right)$ is an indefinite Euclidean space, which is denoted by $\mathbf{R}_{p}^{n+p}$.

Let $S_{p}^{n+p}(c)$ for $c>0$ be the hypersurface in $\mathbf{R}_{p}^{n+p+1}$ given as

$$
\langle x, x\rangle=\frac{1}{c}=: r_{0}^{2} .
$$

Then we know that the $S_{p}^{n+p}(c)$ inherits an indefinite Riemannian metric induced through $\mathbf{R}_{p}^{n+p+1}$ and has constant curvature $c$. This is called a de Sitter space of constant curvature $c$ with index $p$.

On the other hand, let $\mathbf{H}_{p}^{n+p}(c)$ for $c<0$ be the hypersurface in $\mathbf{R}_{p+1}^{n+p+1}$ given as

$$
\langle x, x\rangle=\frac{1}{c}=:-r_{0}^{2} .
$$

* Research partially supported by a Grant-in-Aid for Scientific Research from the Japanese Ministry of Education, Science and Culture and by a Grant-in-Aid for Scientific Research from Josai University. 
Then we also know that the $\mathbf{H}_{p}^{n+p}(c)$ inherits an indefinite Riemannian metric induced through $\mathbf{R}_{p+1}^{n+p+1}$ and has constant curvature $c$. This is called an anti-de Sitter space of constant curvature $c$ with index $p$.

Let $M^{n}$ be an $n$-dimensional Riemannian manifold immersed in $M_{p}^{n+p}(c)$. A submanifold $M^{n}$ of $M_{p}^{n+p}(c)$ is said to be spacelike if the induced metric on $M^{n}$ from that of the ambient space is positive definite.

E. Calabi [1] first studied the Bernstein problem for a maximal spacelike entire graph in the Minkowski space $\mathbf{R}_{1}^{n+1}$ and proved that it has to be hyperplane, when $n \leq 4$. S. Y. Cheng and S. T. Yau [6] proved that the conclusion remains true for all $n$. As a generalization of the Bernstein type problem, T. Ishihara [8] proved that a complete spacelike maximal submanifold $M^{n}$ of $M_{p}^{n+p}(c)(c \geq 0)$ is totally geodesic.

On the other hand, there exist many examples of complete maximal spacelike submanifolds in the anti-de Sitter space $\mathbf{H}_{p}^{n+p}(c)$, which are not totally geodesic. For examples, we consider the following examples.

EXAmPLE 1. We consider the mapping defined by

$$
\begin{gathered}
u_{1}=\frac{1}{\sqrt{-3 c}} y z, u_{2}=\frac{1}{\sqrt{-3 c}} z x, u_{3}=\frac{1}{\sqrt{-3 c}} x y, \\
u_{4}=\frac{1}{2 \sqrt{-3 c}}\left(x^{2}-y^{2}\right), u_{5}=\frac{1}{6 \sqrt{-c}}\left(x^{2}+y^{2}+2 z^{2}\right),
\end{gathered}
$$

where $(x, y, z)$ is the natural coordinate system in $\mathbf{R}_{1}^{3}$ and $\left(u_{1}, u_{2}, u_{3}, u_{4}, u_{5}\right)$ is the natural coordinate system $\mathbf{R}_{3}^{5}$. This defines a complete maximal spacelike isometric immersion of $\mathbf{H}^{2}\left(\frac{c}{3}\right)$ into $\mathbf{H}_{2}^{4}(c)$, where $\mathbf{H}^{n_{i}}\left(c_{i}\right)$ is an $n_{i}$-dimensional hyperbolic space of constant curvature $c_{i}$, which is called the hyperbolic Veronese surface.

EXAmple 2. Let $n_{1}, \cdots, n_{p+1}$ be positive integers and $n=n_{1}+\cdots+n_{p+1}$. Let $x_{i}$ be a point of $\mathbf{H}^{n_{i}}\left(\frac{n c}{n_{i}}\right)$. Then $x=\left(x_{1}, \cdots, x_{p+1}\right)$ is a vector in $\mathbf{R}_{p+1}^{n+p+1}$ with $\langle x, x\rangle=\frac{1}{c}$. This also defines a complete maximal spacelike isometric immersion of $\mathbf{H}^{n_{1}}\left(\frac{n c}{n_{1}}\right) \times \cdots \times \mathbf{H}^{n_{p+1}}\left(\frac{n c}{n_{p+1}}\right)$ into $\mathbf{H}_{p}^{n+p}(c)$.

Hence this case of complete maximal spacelike submanifolds in the anti-de Sitter $\mathbf{H}_{p}^{n+p}(c)$ is very different from the ones in the indefinite Euclidean space $\mathbf{R}_{p}^{n+p}$ and the de Sitter space $S_{p}^{n+p}(c)$. Hence, the investigation of complete maximal spacelike submanifolds in $\mathbf{H}_{p}^{n+p}(c)$ would be very interesting.

T. Ishihara [8] characterized the complete maximal spacelike submanifolds $\mathbf{H}^{n_{1}}\left(\frac{n c}{n_{1}}\right) \times \cdots \times \mathbf{H}^{n_{p+1}}\left(\frac{n c}{n_{p+1}}\right)$ of $\mathbf{H}_{p}^{n+p}(c)$, that is, he proved that let $M^{n}$ be an $n$-dimensional complete maximal spacelike submanifold in $\mathbf{H}_{p}^{n+p}(c)$, then $S \leq-n p c$ and $S=-n p c$ if and only if $M^{n}=\mathbf{H}^{n_{1}}\left(\frac{n c}{n_{1}}\right) \times \cdots \times \mathbf{H}^{n_{p+1}}\left(\frac{n c}{n_{p+1}}\right)$, where $S$ is the squared norm of the second fundamental form of $M^{n}$. When $p=1$, the Bernstein type properties of complete maximal spacelike hypersurfaces in $\mathbf{H}_{1}^{n+1}(c)$ are also studied in [3] and [4].

In particular, if $n=2$, we know that the well known examples of complete maximal spacelike surfaces in the anti-de Sitter space $\mathbf{H}_{2}^{4}(c)$ are the totally geodesic surface $H^{2}(c)$ with $S=0$ and the hyperbolic Veronese surface with $S=\frac{-4 c}{3}$. Therefore, it is natural to ask whether there exist the other complete maximal spacelike surfaces with $S=$ constant in $\mathbf{H}_{2}^{4}(c)$, which are different from the above ones. If 
there exist such surfaces, can we determine all of the value of $S$ ? In this paper we shall answer these problems.

MAIn TheOREM. Let $M^{2}$ be a complete maximal spacelike surface of an anti-de Sitter space $\mathbf{H}_{2}^{4}(c)$ with constant scalar curvature, then $S=0, S=\frac{-10 c}{11}, S=\frac{-4 c}{3}$ or $S=-2 c$, where $S$ is the squared norm of the second fundamental form of $M^{2}$. And

(1) $S=0$ if and only if $M^{2}$ is the totally geodesic surface $\mathbf{H}^{2}(c)$;

(2) $S=\frac{-4 c}{3}$ if and only if $M^{2}$ is the hyperbolic Veronese surface;

(3) $S=-2 c$ if and only if $M^{2}$ is the hyperbolic cylinder of the totally geodesic surface $\mathbf{H}_{1}^{3}(c)$ of $\mathbf{H}_{2}^{4}(c)$.

REMARK 1. It is still open for the author whether there exist complete maximal spacelike surfaces of the anti-de Sitter space $\mathbf{H}_{2}^{4}(c)$ with $S=\frac{-10 c}{11}$.

2. Preliminaries. Let $M^{n}$ be an $n$-dimensional spacelike submanifold of an antide Sitter space $\mathbf{H}_{p}^{n+p}(c)$ of dimension $n+p$ and with index $p$. We choose a local orthonormal frame field $e_{1}, \cdots, e_{n}, e_{n+1}, \cdots, e_{n+p}$ in $\mathbf{H}_{p}^{n+p}(c)$, restricted to $M^{n}$, so that $e_{1}, \cdots, e_{n}$ are tangent to $M^{n}$. With respect to the above frame field of $\mathbf{H}_{p}^{n+p}(c)$, let $\omega_{1}, \cdots, \omega_{n+p}$ denote the dual coframe field. Then

$$
\omega_{\alpha}=0 \quad \text { for any } \quad \alpha=n+1, \cdots, n+p .
$$

It follows from Cartan's Lemma that

$$
\omega_{\alpha i}=\sum_{j} h_{i j}^{\alpha} \omega_{j}, \quad h_{i j}^{\alpha}=h_{j i}^{\alpha}
$$

The structure equations of $M^{n}$ are given by

$$
\begin{aligned}
& \left\{\begin{array}{l}
d \omega_{i}+\sum_{j} \omega_{i j} \wedge \omega_{j}=0, \quad w_{i j}+\omega_{j i}=0, \\
d \omega_{i j}+\sum_{k} \omega_{i k} \wedge \omega_{k j}=\Omega_{i j}, \\
\Omega_{i j}=\frac{1}{2} \sum_{k, l} R_{i j k l} \omega_{k} \wedge \omega_{l},
\end{array}\right. \\
& R_{i j k l}=c\left(\delta_{i k} \delta_{j l}-\delta_{i l} \delta_{j k}\right)-\sum_{\beta}\left(h_{i k}^{\beta} h_{j l}^{\beta}-h_{i l}^{\beta} h_{j k}^{\beta}\right),
\end{aligned}
$$

where $\Omega_{i j}$ (resp. $R_{i j k l}$ ) denotes the curvature form (resp. the components of the curvature tensor) of $M^{n}$.

We have also the structure equations of the normal bundle of $M^{n}$.

$$
\left\{\begin{array}{l}
d \omega_{\alpha \beta}+\sum_{\gamma} \omega_{\alpha \gamma} \wedge \omega_{\gamma \beta}=\Omega_{\alpha \beta}, \\
\Omega_{\alpha \beta}=\frac{1}{2} \sum_{k, l} R_{\alpha \beta k l} \omega_{k} \wedge \omega_{l},
\end{array}\right.
$$




$$
R_{\alpha \beta k l}=-\sum_{t}\left(h_{t k}^{\alpha} h_{t l}^{\beta}-h_{t l}^{\alpha} h_{t k}^{\beta}\right)
$$

The second fundamental form $\mathbf{h}$ of $M^{n}$ is given by

$$
\mathbf{h}=\sum_{i, j, \alpha} h_{i j}^{\alpha} w_{i} w_{j} e_{\alpha}
$$

We recall $\frac{1}{n} \sum_{\alpha}\left(\sum_{i} h_{i i}^{\alpha}\right) e_{\alpha}$ the mean curvature vector. If $\sum_{i} h_{i i}^{\alpha}=0$ for all $\alpha$, then $M^{n}$ is said to be maximal. The Codazzi equation and Ricci formulas for the second fundamental form and its covariant derivatives are given by

$$
\begin{gathered}
h_{i j k}^{\alpha}=h_{i k j}^{\alpha}=h_{j i k}^{\alpha}, \\
h_{i j k l}^{\alpha}-h_{i j l k}^{\alpha}=\sum_{m} h_{i m}^{\alpha} R_{m j k l}+\sum_{m} h_{m j}^{\alpha} R_{m i k l}-\sum_{\beta} h_{i j}^{\beta} R_{\alpha \beta k l}, \\
h_{i j k l m}^{\alpha}-h_{i j k m l}^{\alpha}=\sum_{r} h_{r j k}^{\alpha} R_{r i l m}+\sum_{r} h_{i r k}^{\alpha} R_{r j l m}+\sum_{r} h_{i j r}^{\alpha} R_{r k l m}-\sum_{\beta} h_{i j k}^{\beta} R_{\alpha \beta l m},
\end{gathered}
$$

where $h_{i j k}^{\alpha}, h_{i j k l}^{\alpha}$ and $h_{i j k l m}^{\alpha}$ are the coefficients of the first, the second and the third covariant derivatives of the second fundamental form of $M^{n}$, respectively. If $M^{n}$ is maximal, the scalar curvature is given by

$$
R=n(n-1) c+\sum_{i, j, \alpha}\left(h_{i j}^{\alpha}\right)^{2} .
$$

Hence the scalar curvature is constant if and only if $S=\sum_{i, j, \alpha}\left(h_{i j}^{\alpha}\right)^{2}$ is constant.

The following Generalized Maximum Principle due to Omori [9] and Yau [12] will be used in this paper.

Generalized Maximum Principle (cf. Omori [9] and Yau [12]). Let $M^{n}$ be an n-dimensional complete Riemannian manifold whose Ricci curvature is bounded from below. Let $F$ be a $C^{2}$-function bounded from above on $M^{n}$, then there exists a sequence $\left\{p_{m}\right\}$ of points in $M^{n}$ such that

$$
\lim _{m \rightarrow \infty} F\left(p_{m}\right)=\sup F, \quad \lim _{m \rightarrow \infty}|\nabla F|\left(p_{m}\right)=0, \quad \lim _{m \rightarrow \infty} \sup \Delta F\left(p_{m}\right) \leq 0 .
$$

3. Proof of main theorem. In this section, we assume $n=p=2$. We first compute some local formulas in order to prove Main Theorem. Let $S_{3}:=\sum_{i j}\left(h_{i j}^{3}\right)^{2}$ and $S_{4}:=\sum_{i j}\left(h_{i j}^{4}\right)^{2}$. We know that $S_{3} S_{4}$ is a function defined globally on $M^{2}$. For arbitrary fixed point $p$ in $M^{2}$ we can choose $e_{1}$ and $e_{2}$ such that

$$
h_{i j}^{3}=\lambda_{i} \delta_{i j}
$$

Since $M$ is maximal we get $\lambda_{1}=-\lambda_{2}=: \lambda$. Let

$$
S_{\alpha \beta}=\sum_{i, j} h_{i j}^{\alpha} h_{i j}^{\beta}
$$


We know that the $(2 \times 2)$-matrix $\left(S_{\alpha \beta}\right)$ is symmetric. Hence we can assume that it is diagonal for a suitable choice of $e_{3}$ and $e_{4}$. Thus setting $\mu:=h_{11}^{4}=-h_{22}^{4}$ and $\mu_{1}=h_{12}^{4}$, we have

$$
\sum_{i, j} h_{i j}^{3} h_{i j}^{4}=2 \lambda \mu=0
$$

Theorem 3.1. For $\alpha=3$, 4, we have

$$
\begin{gathered}
\Delta h_{i j}^{\alpha}=(S+2 c) h_{i j}^{\alpha}-2 \sum_{l, t, \beta \neq \alpha} h_{l t}^{\alpha} h_{t j}^{\beta} h_{i l}^{\beta}+\sum_{l, t, \beta \neq \alpha} h_{t l}^{\alpha} h_{t l}^{\beta} h_{i j}^{\beta}, \\
\frac{1}{2} \Delta S=\sum_{i, j, k, \alpha}\left(h_{i j k}^{\alpha}\right)^{2}+(S+2 c) S+2 S_{3} S_{4}, \\
\frac{1}{2} \Delta S_{3}=\sum_{i, j, k}\left(h_{i j k}^{3}\right)^{2}+(S+2 c) S_{3}+S_{3} S_{4},
\end{gathered}
$$

where $S=S_{3}+S_{4}=\sum_{i, j, \alpha}\left(h_{i j}^{\alpha}\right)^{2}$ is the squared norm of the second fundamental form of $M^{2}$ and $S_{3}=\sum_{i, j}\left(h_{i j}^{3}\right)^{2}$ and $S_{4}=\sum_{i, j}\left(h_{i j}^{4}\right)^{2}$.

Proof. For any $\alpha$,

$$
\begin{aligned}
& \Delta h_{i j}^{\alpha}=\sum_{l} h_{i j l}^{\alpha}=\sum_{l} h_{l i j l}^{\alpha} \\
& =\sum_{l} h_{l i l j}^{\alpha}+\sum_{l, t} h_{t i}^{\alpha} R_{t l j l}+\sum_{l, t} h_{l t}^{\alpha} R_{t i j l}-\sum_{l, \beta} h_{l i}^{\beta} R_{\alpha \beta j l} \\
& =\sum_{l, t} h_{t i}^{\alpha}\left[c\left(\delta_{t j} \delta_{l l}-\delta_{t l} \delta_{l j}\right)-\sum_{\beta}\left(h_{t j}^{\beta} h_{l l}^{\beta}-h_{t l}^{\beta} h_{l j}^{\beta}\right)\right] \\
& +\sum_{l, t} h_{t l}^{\alpha}\left[c\left(\delta_{t j} \delta_{i l}-\delta_{t l} \delta_{i j}\right)-\sum_{\beta}\left(h_{t j}^{\beta} h_{i l}^{\beta}-h_{t l}^{\beta} h_{i j}^{\beta}\right)\right] \\
& +\sum_{t, l, \beta} h_{l i}^{\beta}\left(h_{t j}^{\alpha} h_{t l}^{\beta}-h_{t l}^{\alpha} h_{t j}^{\beta}\right) \\
& =(2 c+S) h_{i j}^{\alpha}-2 \sum_{l, t, \beta \neq \alpha} h_{l t}^{\alpha} h_{t j}^{\beta} h_{i l}^{\beta}+\sum_{l, t, \beta \neq \alpha} h_{t l}^{\alpha} h_{t l}^{\beta} h_{i j}^{\beta}, \\
& \frac{1}{2} \Delta S=\sum_{i, j, k, \alpha}\left(h_{i j k}^{\alpha}\right)^{2}+\sum_{i, j, \alpha} h_{i j}^{\alpha} \Delta h_{i j}^{\alpha} \\
& =\sum_{i, j, k, \alpha}\left(h_{i j k}^{\alpha}\right)^{2}+(2 c+S) S+2 S_{3} S_{4}, \\
& \frac{1}{2} \Delta S_{3}=\sum_{i, j, k}\left(h_{i j k}^{3}\right)^{2}+\sum_{i, j} h_{i j}^{3} \Delta h_{i j}^{3} \\
& =\sum_{i, j, k}\left(h_{i j k}^{3}\right)^{2}+(2 c+S) S_{3}+S_{3} S_{4} .
\end{aligned}
$$


This finishes the Proof of Theorem 3.1.

Theorem 3.2.

$$
\begin{aligned}
& \frac{1}{2} \Delta \sum_{i, j, k, \alpha}\left(h_{i j k}^{\alpha}\right)^{2} \\
& =\sum_{i, j, k, l, \alpha}\left(h_{i j k l}^{\alpha}\right)^{2}+\left(\frac{9}{2} S+7 c\right) \sum_{i, j, k, \alpha}\left(h_{i j k}^{\alpha}\right)^{2} \\
& +3|\nabla S|^{2}-5 \sum_{\alpha} S_{\alpha} \sum_{i, j, k}\left(h_{i j k}^{\alpha}\right)^{2}
\end{aligned}
$$

Since $M^{2}$ is maximal, for any $\alpha$, we have

$$
h_{11}^{\alpha}+h_{22}^{\alpha}=0 \text {. }
$$

Hence

$$
h_{11 l}^{\alpha}=-h_{22 l}^{\alpha}, \quad h_{11 l k}^{\alpha}=-h_{22 l k}^{\alpha} \quad \text { for any } l, k .
$$

In the sequel, we will often use the formula (3.7).

Proof.

$$
\begin{aligned}
& \sum_{i, j, k, \alpha} h_{i j k}^{\alpha} \Delta h_{i j k}^{\alpha}=\sum_{i, j, k, l, \alpha} h_{i j k}^{\alpha} h_{i j k l l}^{\alpha} \\
& =\sum_{i, j, k, l, \alpha} h_{i j k}^{\alpha}\left[h_{i j l k l}^{\alpha}+\nabla_{l}\left(\sum_{t} h_{t j}^{\alpha} R_{t i k l}\right.\right. \\
& \left.\left.+\sum_{t} h_{t i}^{\alpha} R_{t j k l}-\sum_{\beta} h_{i j}^{\beta} R_{\alpha \beta k l}\right)\right] \\
& =\sum_{i, j, k, l, \alpha} h_{i j k}^{\alpha}\left[h_{i j l l k}^{\alpha}+\sum_{t} h_{t j l}^{\alpha} R_{t i k l}\right. \\
& \left.+\sum_{t} h_{t i l}^{\alpha} R_{t j k l}+\sum_{t} h_{i j t}^{\alpha} R_{t l k l}-\sum_{\beta} h_{i j l}^{\beta} R_{\alpha \beta k l}\right] \\
& +\sum_{i, j, k, l, \alpha} h_{i j k}^{\alpha}\left[\sum_{t} h_{t j l}^{\alpha} R_{t i k l}+\sum_{t} h_{t i l}^{\alpha} R_{t j k l}-\sum_{\beta} h_{i j l}^{\beta} R_{\alpha \beta k l}\right] \\
& +\sum_{i, j, k, l, \alpha} h_{i j k}^{\alpha}\left[\sum_{t} h_{t j}^{\alpha} \nabla_{l} R_{t i k l}+\sum_{t} h_{t i}^{\alpha} \nabla_{l} R_{t j k l}-\sum_{\beta} h_{i j}^{\beta} \nabla_{l} R_{\alpha \beta k l}\right] \\
& =\sum_{i, j, k, \alpha} h_{i j k}^{\alpha}\left\{\nabla _ { k } \left[(S+2 c) h_{i j}^{\alpha}\right.\right. \\
& \left.\left.-2 \sum_{l, t, \beta \neq \alpha} h_{l t}^{\alpha} h_{t j}^{\beta} h_{i l}^{\beta}+\sum_{l, t, \beta \neq \alpha} h_{t l}^{\alpha} h_{t l}^{\beta} h_{i j]}^{\beta}\right]\right\} \\
& +2 \sum_{i, j, k, l, t, \alpha} h_{i j k}^{\alpha} h_{t j l}^{\alpha} R_{t i k l}+2 \sum_{i, j, k, l, t, \alpha} h_{i j k}^{\alpha} h_{t i l}^{\alpha} R_{t j k l} \\
& +\sum_{i, j, k, l, t, \alpha} h_{i j k}^{\alpha} h_{i j t}^{\alpha} R_{t l k l}-2 \sum_{i, j, k, l, \alpha, \beta} h_{i j k}^{\alpha} h_{i j l}^{\beta} R_{\alpha \beta k l} \\
& +\sum_{i, j, k, l, \alpha} h_{i j k}^{\alpha}\left[\sum_{t} h_{t j}^{\alpha} \nabla_{l} R_{t i k l}+\sum_{t} h_{t i}^{\alpha} \nabla_{l} R_{t j k l}-\sum_{\beta} h_{i j}^{\beta} \nabla_{l} R_{\alpha \beta k l}\right] .
\end{aligned}
$$




$$
\begin{aligned}
& \sum_{i, j, k, \alpha} h_{i j k}^{\alpha}\left\{\nabla_{k}\left[(S+2 c) h_{i j}^{\alpha}-2 \sum_{l, t, \beta \neq \alpha} h_{l t}^{\alpha} h_{t j}^{\beta} h_{i l}^{\beta}+\sum_{l, t, \beta \neq \alpha} h_{t l}^{\alpha} h_{t l}^{\beta} h_{i j}^{\beta}\right]\right\} \\
& =(S+2 c) \sum_{i, j, k, \alpha}\left(h_{i j k}^{\alpha}\right)^{2}+\sum_{i, j, k, \alpha} h_{i j}^{\alpha} h_{i j k}^{\alpha} \nabla_{k} S \\
& -2 \sum_{i, j, k, t, l, \alpha, \beta \neq \alpha} h_{i j k}^{\alpha} h_{t l k}^{\alpha} h_{t j}^{\beta} h_{i l}^{\beta}-2 \sum_{i, j, k, t, l, \alpha, \beta \neq \alpha} h_{i j k}^{\alpha} h_{t l}^{\alpha} h_{t j k}^{\beta} h_{i l}^{\beta} \\
& -2 \sum_{i, j, k, t, l, \alpha, \beta \neq \alpha} h_{i j k}^{\alpha} h_{t l}^{\alpha} h_{t j}^{\beta} h_{i l k}^{\beta}+\sum_{i, j, k, t, l, \alpha, \beta \neq \alpha} h_{i j k}^{\alpha} h_{t l k}^{\alpha} h_{t l}^{\beta} h_{i j}^{\beta} \\
& +\sum_{i, j, k, t, l, \alpha, \beta \neq \alpha} h_{i j k}^{\alpha} h_{t l}^{\alpha} h_{t l k}^{\beta} h_{i j}^{\beta}+\sum_{i, j, k, t, l, \alpha, \beta \neq \alpha} h_{i j k}^{\alpha} h_{i j k}^{\beta} h_{t l}^{\alpha} h_{t l}^{\beta} \\
& =(S+2 c) \sum_{i, j, k, \alpha}\left(h_{i j k}^{\alpha}\right)^{2}+\frac{1}{2}|\nabla S|^{2}-2 \sum_{i, j, k, l, t} h_{i j k}^{3} h_{t l k}^{3} h_{t j}^{4} h_{i l}^{4} \\
& -4 \sum_{i, j, k, t} h_{i j k}^{4} h_{t j k}^{3} h_{t i}^{4} h_{i i}^{3}-4 \sum_{i, j, k, t} h_{i j k}^{4} h_{t i k}^{3} h_{t j}^{4} h_{j j}^{3}+\sum_{i, j, k, l, t} h_{i j k}^{3} h_{t l k}^{3} h_{t l}^{4} h_{i j}^{4} \\
& +\frac{S_{3}}{2} \sum_{i, j, k}\left(h_{i j k}^{4}\right)^{2}+4 \lambda \sum_{i, j, k} h_{11 k}^{4} h_{i j}^{4} h_{i j k}^{3}(b y(3.2)) \\
& =(S+2 c) \sum_{i, j, k, \alpha}\left(h_{i j k}^{\alpha}\right)^{2}+\frac{1}{2}|\nabla S|^{2}+\frac{S_{3}}{2} \sum_{i, j, k}\left(h_{i j k}^{4}\right)^{2} \\
& +2 \sum_{i, j, k, l, t} h_{i j k}^{3} h_{t l k}^{3} h_{t j}^{4} h_{i l}^{4}-8 \sum_{i, j, k, t} h_{i j k}^{4} h_{t j k}^{3} h_{t i}^{4} h_{i i}^{3} \\
& +\sum_{k}\left(\sum_{i, j} h_{i j k}^{3} h_{i j}^{4}\right)^{2}+4 \lambda \sum_{i, j, k} h_{11 k}^{4} h_{i j}^{4} h_{i j k}^{3} . \\
& \\
& +
\end{aligned}
$$

$$
\begin{aligned}
& \sum_{i, j, k, l, t, \alpha} h_{i j k}^{\alpha} h_{i j t}^{\alpha} R_{t l k l} \\
= & \sum_{i, j, k, l, t, \alpha} h_{i j k}^{\alpha} h_{i j t}^{\alpha}\left[c\left(\delta_{t k} \delta_{l l}-\delta_{t l} \delta_{l k}\right)-\sum_{\beta}\left(h_{t k}^{\beta} h_{l l}^{\beta}-h_{t l}^{\beta} h_{l k}^{\beta}\right)\right] \\
= & \left(\frac{S}{2} c\right) \sum_{i, j, k, \alpha}\left(h_{i j k}^{\alpha}\right)^{2} .
\end{aligned}
$$

$$
\begin{aligned}
& -2 \sum_{i, j, k, l, \alpha, \beta} h_{i j k}^{\alpha} h_{i j l}^{\beta} R_{\alpha \beta k l} \\
& =2 \sum_{i, j, k, l, \alpha, \beta} h_{i j k}^{\alpha} h_{i j l}^{\beta} \sum_{t}\left(h_{t k}^{\alpha} h_{t l}^{\beta}-h_{t l}^{\alpha} h_{t k}^{\beta}\right) \\
& =-4 \sum_{i, j, k, l} h_{i j k}^{3} h_{i j l}^{4} h_{l k}^{4}\left(h_{l l}^{3}-h_{k k}^{3}\right) .
\end{aligned}
$$




$$
\begin{aligned}
& 2 \sum_{i, j, k, l, t, \alpha} h_{i j k}^{\alpha} h_{t j l}^{\alpha} R_{t i k l}+2 \sum_{i, j, k, l, t, \alpha} h_{i j k}^{\alpha} h_{t i l}^{\alpha} R_{t j k l} \\
& =2 \sum_{i, j, k, l, t, \alpha} h_{i j k}^{\alpha} h_{t j l}^{\alpha}\left[c\left(\delta_{t k} \delta_{i l}-\delta_{t l} \delta_{i k}\right)-\sum_{\beta}\left(h_{t k}^{\beta} h_{i l}^{\beta}-h_{t l}^{\beta} h_{i k}^{\beta}\right)\right] \\
& +2 \sum_{i, j, k, l, t, \alpha} h_{i j k}^{\alpha} h_{i t l}^{\alpha}\left[c\left(\delta_{t k} \delta_{j l}-\delta_{t l} \delta_{j k}\right)-\sum_{\beta}\left(h_{t k}^{\beta} h_{j l}^{\beta}-h_{t l}^{\beta} h_{j k}^{\beta}\right)\right] \\
& =4 c \sum_{i, j, k, \alpha}\left(h_{i j k}^{\alpha}\right)^{2}-4 \sum_{i, j, k, l, t, \alpha, \beta} h_{i j k}^{\alpha} h_{t j l}^{\alpha} h_{t k}^{\beta} h_{i l}^{\beta} \\
& +4 \sum_{i, j, k, l, t, \alpha, \beta} h_{i j k}^{\alpha} h_{t j l}^{\alpha} h_{t l}^{\beta} h_{i k}^{\beta} \\
& =4 c \sum_{i, j, k, \alpha}\left(h_{i j k}^{\alpha}\right)^{2}+2 S_{3} \sum_{i, j, k, \alpha}\left(h_{i j k}^{\alpha}\right)^{2} \\
& -4 \sum_{i, j, k, t, l} h_{i j k}^{3} h_{t j l}^{3}\left(h_{t k}^{4} h_{i l}^{4}-h_{t l}^{4} h_{i k}^{4}\right) \\
& -4 \sum_{i, j, k, t, l} h_{i j k}^{4} h_{t j l}^{4}\left(h_{t k}^{4} h_{i l}^{4}-h_{t l}^{4} h_{i k}^{4}\right) .
\end{aligned}
$$

$$
\begin{aligned}
& \sum_{i, j, k, l, \alpha} h_{i j k}^{\alpha}\left[\sum_{t} h_{t j}^{\alpha} \nabla_{l} R_{t i k l}+\sum_{t} h_{t i}^{\alpha} \nabla_{l} R_{t j k l}\right. \\
& \left.-\sum_{\beta} h_{i j}^{\beta} \nabla_{l} R_{\alpha \beta k l}\right] \\
& =-\sum_{i, j, k, t, l, \alpha, \beta} h_{i j k}^{\alpha} h_{t j}^{\alpha} \nabla_{l}\left(h_{t k}^{\beta} h_{i l}^{\beta}-h_{t l}^{\beta} h_{i k}^{\beta}\right) \\
& -\sum_{i, j, k, t, l, \alpha, \beta} h_{i j k}^{\alpha} h_{t i}^{\alpha} \nabla_{l}\left(h_{t k}^{\beta} h_{j l}^{\beta}-h_{t l}^{\beta} h_{j k}^{\beta}\right) \\
& +\sum_{i, j, k, t, l, \alpha, \beta} h_{i j k}^{\alpha} h_{i j}^{\beta} \nabla_{l}\left(h_{t k}^{\alpha} h_{t l}^{\beta}-h_{t l}^{\alpha} h_{t k}^{\beta}\right) \\
& =-2 \sum_{i, j, k, t, l, \alpha, \beta} h_{i j k}^{\alpha} h_{t j}^{\alpha}\left(h_{t k l}^{\beta} h_{i l}^{\beta}-h_{t l}^{\beta} h_{i k l}^{\beta}\right) \\
& +\sum_{i, j, k, t, l, \alpha, \beta} h_{i j k}^{\alpha} h_{i j}^{\beta}\left(h_{t k l}^{\alpha} h_{t l}^{\beta}-h_{t l}^{\alpha} h_{t k l}^{\beta}\right) \\
& =S_{3} \sum_{i, j, k}\left(h_{i j k}^{3}\right)^{2}+\frac{S_{3}}{2} \sum_{i, j, k}\left(h_{i j k}^{4}\right)^{2} \\
& +\sum_{k}\left(\sum_{i, j} h_{i j}^{4} h_{i j k}^{3}\right)^{2}-4 \lambda \sum_{i, j, k} h_{11 k}^{4} h_{i j}^{4} h_{i j k}^{3} \\
& -2 \sum_{i, j, k, t, l} h_{i j k}^{4} h_{t k l}^{4} h_{i l}^{4} h_{t j}^{4}+2 \sum_{i, j, k, t, l} h_{i j k}^{4} h_{i k l}^{4} h_{t l}^{4} h_{t j}^{4} \\
& -4 \sum_{i, j, k, t, l} h_{i j k}^{3} h_{i k l}^{4} h_{j l}^{4}\left(h_{i i}^{3}-h_{j j}^{3}\right) \\
&
\end{aligned}
$$

Hence, (3.8) (3.13) yield 


$$
\begin{aligned}
& \sum_{i, j, k, \alpha} h_{i j k}^{\alpha} \Delta h_{i j k}^{\alpha} \\
& =\left(\frac{3}{2} S+7 c\right) \sum_{i, j, k, \alpha}\left(h_{i j k}^{\alpha}\right)^{2}+\frac{1}{2}|\nabla S|^{2}+3 S_{3} \sum_{i, j, k, \alpha}\left(h_{i j k}^{\alpha}\right)^{2} \\
& -4 \sum_{i, j, k, l} h_{i j k}^{3} h_{i j l}^{4} h_{l k}^{4}\left(h_{l l}^{3}-h_{k k}^{3}\right) \\
& -4 \sum_{i, j, k, l} h_{i j k}^{3} h_{i k l}^{4} h_{j l}^{4}\left(h_{i i}^{3}-h_{j j}^{3}\right) \\
& -2 \sum_{i, j, k, l, t} h_{i j k}^{3} h_{t l k}^{3} h_{t j}^{4} h_{i l}^{4}-8 \sum_{i, j, k, t} h_{i j k}^{4} h_{t j k}^{3} h_{t i}^{4} h_{i i}^{3} \\
& -4 \sum_{i, j, k, t, l} h_{i j k}^{3} h_{t j l}^{3}\left(h_{t k}^{4} h_{i l}^{4}-h_{t l}^{4} h_{i k}^{4}\right) \\
& -4 \sum_{i, j, k, t, l} h_{i j k}^{4} h_{t j l}^{4}\left(h_{t k}^{4} h_{i l}^{4}-h_{t l}^{4} h_{i k}^{4}\right) \\
& -2 \sum_{i, j, k, t, l} h_{i j k}^{4} h_{t k l}^{4} h_{i l}^{4} h_{t j}^{4}+2 \sum_{i, j, k, t, l} h_{i j k}^{4} h_{i k l}^{4} h_{t l}^{4} h_{t j}^{4} \\
& +2 \sum_{k}\left(\sum_{i, j} h_{i j k}^{3} h_{i j}^{4}\right)^{2} .
\end{aligned}
$$

$\left.-h_{2 j k}^{4} h_{1 j k}^{3} \mu_{1}+h_{2 j k}^{4} h_{2 j k}^{3} \mu\right)$

$=8 \lambda \mu_{1}\left(h_{1 j k}^{4} h_{2 j k}^{3}-h_{2 j k}^{4} h_{1 j k}^{3}\right) \quad($ by (3.2))

$=32 \lambda \mu_{1}\left(h_{222}^{4} h_{111}^{3}-h_{111}^{4} h_{222}^{3}\right)$.

$$
\begin{aligned}
& 4 \sum_{i, j, k, l} h_{i j k}^{3} h_{i j l}^{4} h_{l k}^{4}\left(h_{l l}^{3}-h_{k k}^{3}\right) \\
& =4 \sum_{i, j} h_{i j 1}^{3} h_{i j 2}^{4} h_{12}^{4}\left(h_{22}^{3}-h_{11}^{3}\right) \\
& +4 \sum_{i, j} h_{i j 2}^{3} h_{i j 1}^{4} h_{12}^{4}\left(h_{11}^{3}-h_{22}^{3}\right) \\
& =-8 \lambda \mu_{1} \sum_{i j}\left(h_{1 i j}^{3} h_{2 i j}^{4}-h_{1 i j}^{4} h_{2 i j}^{3}\right) \quad \text { (by (3.2)) } \\
& =32 \lambda \mu_{1}\left(h_{222}^{4} h_{111}^{3}-h_{111}^{4} h_{222}^{3}\right) .
\end{aligned}
$$




$$
\begin{aligned}
& 4 \sum_{i, j, k, l} h_{i j k}^{3} h_{i k l}^{4} h_{l j}^{4}\left(h_{i i}^{3}-h_{j j}^{3}\right) \\
& =4 \sum_{i, j,} h_{12 k}^{3} h_{1 k l}^{4} h_{l 2}^{4}\left(h_{11}^{3}-h_{22}^{3}\right) \\
& +4 \sum_{i, j,} h_{21 k}^{3} h_{2 k l}^{4} h_{1 l}^{4}\left(h_{22}^{3}-h_{11}^{3}\right) \\
& =8 \lambda \sum_{k, l}\left(h_{12 k}^{3} h_{1 k l}^{4} h_{l 2}^{4}-h_{12 k}^{3} h_{2 k l}^{4} h_{1 l}^{4}\right) \\
& =16 \lambda \mu_{1}\left(h_{222}^{4} h_{111}^{3}-h_{111}^{4} h_{222}^{3}\right) \quad \text { (by (3.2)). }
\end{aligned}
$$

$$
\begin{aligned}
& 4 \sum_{i, j, k, t, l, \alpha} h_{i j k}^{\alpha} h_{t j l}^{\alpha}\left(h_{t k}^{4} h_{i l}^{4}-h_{t l}^{4} h_{i k}^{4}\right) \\
& =4 \sum_{i, j, k, t, \alpha} h_{i j k}^{\alpha} h_{t j 1}^{\alpha}\left(h_{t k}^{4} h_{i 1}^{4}-h_{t 1}^{4} h_{i k}^{4}\right) \\
& +4 \sum_{i, j, k, t, \alpha} h_{i j k}^{\alpha} h_{t j 2}^{\alpha}\left(h_{t k}^{4} h_{i 2}^{4}-h_{t 2}^{4} h_{i k}^{4}\right) \\
& =4 \sum_{i, j, k, \alpha} h_{i j k}^{\alpha} h_{1 j 1}^{\alpha}\left(h_{1 k}^{4} h_{i 1}^{4}-h_{11}^{4} h_{i k}^{4}\right) \\
& +4 \sum_{i, j, k, t, \alpha} h_{i j k}^{\alpha} h_{1 j 2}^{\alpha}\left(h_{1 k}^{4} h_{i 2}^{4}-h_{12}^{4} h_{i k}^{4}\right) \\
& +4 \sum_{i, j, k, \alpha} h_{i j k}^{\alpha} h_{2 j 1}^{\alpha}\left(h_{2 k}^{4} h_{i 1}^{4}-h_{21}^{4} h_{i k}^{4}\right) \\
& +4 \sum_{i, j, k, \alpha} h_{i j k}^{\alpha} h_{2 j 2}^{\alpha}\left(h_{2 k}^{4} h_{i 2}^{4}-h_{22}^{4} h_{i k}^{4}\right) \\
& =-8 \sum_{i, j, k, \alpha} h_{i j k}^{\alpha} h_{11 j}^{\alpha} h_{i k}^{4} h_{11}^{4} \\
& +4 \sum_{i, j, k, \alpha} h_{i j k}^{\alpha} h_{1 j 1}^{\alpha}\left(h_{1 k}^{4} h_{i 1}^{4}-h_{2 k}^{4} h_{2 i}^{4}\right) \\
& -8 \sum_{i, j, k, \alpha} h_{i j k}^{\alpha} h_{12 j}^{\alpha} h_{i k}^{4} h_{12}^{4}+8 \sum_{i, j, k, \alpha} h_{i j k}^{\alpha} h_{12 j}^{\alpha} h_{1 i}^{4} h_{2 k}^{4} \\
& =-2 S_{4} \sum_{i, j, k, \alpha}\left(h_{i j k}^{\alpha}\right)^{2} .
\end{aligned}
$$

For any $\alpha$,

$$
\begin{aligned}
& 2 \sum_{i, j, k, l, t} h_{i j k}^{\alpha} h_{t l k}^{\alpha} h_{t j}^{4} h_{i l}^{4} \\
& =2 \sum_{i, j, k, l} h_{i j k}^{\alpha} h_{1 l k}^{\alpha} h_{1 j}^{4} h_{i l}^{4}+2 \sum_{i, j, k, l, t} h_{i j k}^{\alpha} h_{2 l k}^{\alpha} h_{2 j}^{4} h_{i l}^{4} \\
& =2 \mu \sum_{i, k, l}\left(h_{1 i k}^{\alpha} h_{1 l k}^{\alpha}-h_{2 i k}^{\alpha} h_{2 l k}^{\alpha}\right) h_{i l}^{4}+4 \mu_{1} \sum_{i, k, l} h_{1 i k}^{\alpha} h_{2 l k}^{\alpha} h_{i l}^{4} \\
& =0 .
\end{aligned}
$$




$$
\begin{aligned}
& 2 \sum\left(\sum_{i, j} h_{i j k}^{3} h_{i j}^{4}\right)^{2} \\
&= 2 \sum_{k}\left(\sum_{i j} h_{i j}^{4} h_{i j k}^{3}\right)^{2}=S_{4} \sum_{i, j, k}\left(h_{i j k}^{3}\right)^{2} . \\
& 2 \sum_{i, j, k, t, l} h_{i j k}^{4} h_{i k l}^{4} h_{t l}^{4} h_{t j}^{4} \\
&=2 \sum_{i, j, k, t} h_{i j k}^{4} h_{i k 1}^{4} h_{t 1}^{4} h_{t j}^{4}+2 \sum_{i, j, k, t} h_{i j k}^{4} h_{i k 2}^{4} h_{t 2}^{4} h_{t j}^{4} \\
&=S_{4} \sum_{i, j, k}\left(h_{i j k}^{4}\right)^{2} .
\end{aligned}
$$

According to $(3.14) \sim(3.21)$, we get

$$
\begin{aligned}
& \sum_{i, j, k, \alpha} h_{i j k}^{\alpha} \Delta h_{i j k}^{\alpha} \\
& =\left(\frac{9}{2} S+7 c\right) \sum_{i, j, k, \alpha}\left(h_{i j k}^{\alpha}\right)^{2}+\frac{1}{2}|\nabla S|^{2} \\
& -80 \lambda \mu_{1}\left(h_{111}^{3} h_{222}^{4}-h_{111}^{4} h_{222}^{3}\right) .
\end{aligned}
$$

Since, for any $\alpha$,

$$
\sum_{i, j, k}\left(h_{i j k}^{\alpha}\right)^{2}=4\left\{\left(h_{111}^{\alpha}\right)^{2}+\left(h_{222}^{\alpha}\right)^{2}\right\}
$$

and

$$
\begin{aligned}
& |\nabla S|^{2}=\sum_{l}\left(\nabla_{l} S\right)^{2} \\
& =\sum_{l}\left(2 \sum_{i, j, \alpha} h_{i j}^{\alpha} h_{i j l}^{\alpha}\right)^{2} \\
& =16 \sum_{l}\left(\lambda h_{11 l}^{3}+\mu h_{11 l}^{4}+\mu_{1} h_{12 l}^{4}\right)^{2} \\
& =2 \sum_{i, j, k, \alpha} S_{\alpha}\left(h_{i j k}^{\alpha}\right)^{2}-32 \lambda \mu_{1}\left(h_{111}^{3} h_{222}^{4}-h_{111}^{4} h_{222}^{3}\right),
\end{aligned}
$$

we obtain

$$
-8 \lambda \mu_{1}\left(h_{111}^{3} h_{222}^{4}-h_{111}^{4} h_{222}^{3}\right)=\frac{1}{4}|\nabla S|^{2}-\frac{1}{2} \sum_{i, j, k, \alpha} S_{\alpha}\left(h_{i j k}^{\alpha}\right)^{2}
$$

From

$$
\frac{1}{2} \Delta \sum_{i, j, k, \alpha}\left(h_{i j k}^{\alpha}\right)^{2}=\sum_{i, j, k, \alpha} h_{i j k}^{\alpha} \Delta h_{i j k}^{\alpha}+\sum_{i, j, k, l, \alpha}\left(h_{i j k l}^{\alpha}\right)^{2},
$$

(3.22) and (3.23) yields the Theorem 3.2. 
Lemma 1.

$$
\begin{aligned}
& \sum_{i, j, k, l, \alpha}\left(h_{i j k l}^{\alpha}\right)^{2}=\sum_{i \neq j, \alpha}\left(h_{i i j j}^{\alpha}-h_{j j i i}^{\alpha}\right)^{2}+\sum_{i \neq j, \alpha}\left(h_{i i j j}^{\alpha}+h_{j j i i}^{\alpha}\right)^{2} \\
& +\sum_{i \neq j, \alpha}\left(h_{i i i j}^{\alpha}-h_{i i j i}^{\alpha}\right)^{2}+\sum_{i \neq j, \alpha}\left(h_{i i i j}^{\alpha}+h_{i i j i}^{\alpha}\right)^{2}
\end{aligned}
$$

Proof.

$$
\sum_{i, j, k, l, \alpha}\left(h_{i j k l}^{\alpha}\right)^{2}=\sum_{i, \alpha}\left(h_{i i i i}^{\alpha}\right)^{2}+3 \sum_{i \neq j, \alpha}\left(h_{i i j j}^{\alpha}\right)^{2}+\sum_{i \neq j, \alpha}\left(h_{i i i j}^{\alpha}\right)^{2}+3 \sum_{i \neq j, \alpha}\left(h_{i i j i}^{\alpha}\right)^{2} .
$$

Since

$$
h_{i i i i}^{\alpha}=-h_{j j i i}^{\alpha} \quad \text { for any } \quad \alpha \text { and } j \neq i
$$

and

$$
h_{i i j}^{\alpha}=-h_{j j i j}^{\alpha} \quad \text { for any } \quad \alpha \text { and } j \neq i,
$$

we know that Lemma 1 holds.

\section{LEMMA 2.}

$$
\begin{gathered}
h_{1122}^{3}-h_{2211}^{3}=\lambda\left(2 c+S+S_{4}\right), \\
h_{1112}^{3}-h_{1121}^{3}=0, \\
h_{1122}^{4}-h_{2211}^{4}=(2 c+S) \mu, \\
h_{1112}^{4}-h_{1121}^{4}=-\left(2 c+S+S_{3}\right) \mu_{1} .
\end{gathered}
$$

Proof. From the Ricci formula (2.8), we have

$$
\begin{aligned}
& h_{i i j j}^{\alpha}-h_{j j i i}^{\alpha}=h_{i j i j}^{\alpha}-h_{i j j i}^{\alpha} \\
& =\sum_{t} h_{t j}^{\alpha} R_{t i i j}+\sum_{t} h_{i t}^{\alpha} R_{t j i j}-\sum_{t} h_{i j}^{\beta} R_{\alpha \beta i j} \\
& =\left(h_{i i}^{\alpha}-h_{j j}^{\alpha}\right) c-\frac{S}{2}\left(h_{j j}^{\alpha}-h_{i i}^{\alpha}\right) \\
& -\sum_{t, \beta \neq \alpha} h_{t j}^{\alpha} h_{t i}^{\beta} h_{i j}^{\beta}+\sum_{t, \beta \neq \alpha} h_{i t}^{\alpha} h_{t j}^{\beta} h_{i j}^{\beta} .
\end{aligned}
$$

Hence

$$
\begin{gathered}
h_{1122}^{3}-h_{2211}^{3}=\lambda\left(2 c+S+S_{4}\right), \\
h_{1122}^{4}-h_{2211}^{4}=(2 c+S) \mu .
\end{gathered}
$$


By the same proof, we can obtain

$$
\begin{gathered}
h_{1112}^{3}-h_{1121}^{3}=2 \lambda \mu \mu_{1}=0 \quad(\text { by }(3.2)), \\
h_{1112}^{4}-h_{1121}^{4}=-\left(2 c+S+S_{3}\right) \mu_{1} .
\end{gathered}
$$

Thus we complete the proof of Lemma 2.

Next, we shall prove the Main Theorem. Since the scalar curvature is constant if and only if $S$ is constant, in the sequel, we assume that $S$ is constant.

Proof of Main Theorem. If $S=0$, then $M$ is totally geodesic because $S$ is constant. Next we assume $S \neq 0$. Since $S$ is constant, we have

$$
\nabla_{l} S=0, \quad \text { for } \quad l=1,2,
$$

namely,

$$
\left\{\begin{array}{l}
2 \lambda h_{11 l}^{3}+2 \mu h_{11 l}^{4}+2 \mu_{1} h_{12 l}^{4}=0 \\
2 \lambda h_{111}^{3}+2 \mu h_{111}^{4}-2 \mu_{1} h_{222}^{4}=0 \\
2 \lambda h_{222}^{3}+2 \mu h_{222}^{4}+2 \mu_{1} h_{111}^{4}=0
\end{array}\right.
$$

Hence we obtain

$$
S_{3} \sum_{i, j, k}\left(h_{i j k}^{3}\right)^{2}=S_{4} \sum_{i, j, k}\left(h_{i j k}^{4}\right)^{2},
$$

from

$$
\sum_{i, j, k}\left(h_{i j k}^{\alpha}\right)^{2}=4\left[\left(h_{111}^{\alpha}\right)^{2}+\left(h_{222}^{\alpha}\right)^{2}\right]
$$

We know that $S_{3} S_{4}$ are function defined globally on $M$. Because $S$ is constant, from Gauss equation, we infer that the sectional curvature is bounded from below and that function $S_{3} S_{4}$ is bounded because $0 \leq S_{3} S_{4} \leq S^{2}$. Since $M$ is complete, from the Generalized Maximum Principle due to Omori and Yau, we know that there exists a sequence $\left\{p_{m}\right\} \subset M^{2}$ of points such that

$$
\begin{gathered}
\lim _{m \rightarrow \infty}\left(S_{3} S_{4}\right)\left(p_{m}\right)=\inf \left(S_{3} S_{4}\right), \\
\lim _{m \rightarrow \infty}\left|\nabla\left(S_{3} S_{4}\right)\right|\left(p_{m}\right)=0, \\
\lim _{m \rightarrow \infty} \inf \Delta\left(S_{3} S_{4}\right)\left(p_{m}\right) \geq 0
\end{gathered}
$$

From Theorem 3.1, we have

$$
\sum_{i, j, k, \alpha}\left(h_{i j k}^{\alpha}\right)^{2}=-S(S+2 c)-2 S_{3} S_{4} .
$$


because $S$ is constant. Hence $\sum_{i, j, k, \alpha}\left(h_{i j k}^{\alpha}\right)^{2}$ is bounded and

$$
\begin{gathered}
\lim _{m \rightarrow \infty} \sum_{i, j, k, \alpha}\left(h_{i j k}^{\alpha}\right)^{2}\left(p_{m}\right)=\sup \sum_{i, j, k, \alpha}\left(h_{i j k}^{\alpha}\right)^{2}, \\
\lim _{m \rightarrow \infty}\left|\nabla \sum_{i, j, k, \alpha}\left(h_{i j k}^{\alpha}\right)^{2}\right|\left(p_{m}\right)=-2 \lim _{m \rightarrow \infty}\left|\nabla\left(S_{3} S_{4}\right)\right|\left(p_{m}\right)=0 .
\end{gathered}
$$

From

$$
\Delta \sum_{i, j, k, \alpha}\left(h_{i j k}^{\alpha}\right)^{2}=-2 S_{3} \Delta S_{4}-4 \nabla S_{3} \cdot \nabla S_{4}-2 S_{4} \Delta S_{3}
$$

and Theorem 3.1 and Theorem 3.2, we obtain that $\sum_{i, j, k, l, \alpha}\left(h_{j j k l}^{\alpha}\right)^{2}$ is bounded. Thus, we can assume $\lim _{m \rightarrow \infty} S_{3}\left(p_{m}\right)=\tilde{S}_{3}, \quad \lim _{m \rightarrow \infty} S_{4}\left(p_{m}\right)=\tilde{S}_{4}, \quad \lim _{m \rightarrow \infty} \lambda\left(p_{m}\right)=\tilde{\lambda}$, $\lim _{m \rightarrow \infty} \mu\left(p_{m}\right)=\tilde{\mu}, \lim _{m \rightarrow \infty} \mu_{1}\left(p_{m}\right)=\tilde{\mu}_{1}, \lim _{m \rightarrow \infty} h_{i j k}^{\alpha}\left(p_{m}\right)=\tilde{h}_{i j k}^{\alpha}$ and $\lim _{m \rightarrow \infty} h_{i j k l}^{\alpha}\left(p_{m}\right)$ $=h_{i j k l}^{\alpha}$, by taking a subsequence if necessary. Since

$$
\lim _{m \rightarrow \infty}\left|\nabla\left(S_{3} S_{4}\right)\right|\left(p_{m}\right)=0
$$

and

$$
\nabla_{l}\left(S_{3} S_{4}\right)=S_{3} \nabla_{l} S_{4}+S_{4} \nabla_{l} S_{3}
$$

we have

$$
\lim _{m \rightarrow \infty}\left(S_{3} \nabla_{l} S_{4}+S_{4} \nabla_{l} S_{3}\right)\left(p_{m}\right)=0 .
$$

From $S=S_{3}+S_{4}$, we get

$$
\nabla_{l} S_{3}=-\nabla_{l} S_{4}
$$

Therefore,

$$
\lim _{m \rightarrow \infty}\left(S_{3}-S_{4}\right)\left(\nabla_{l} S_{4}\right)\left(p_{m}\right)=\lim _{m \rightarrow \infty}\left(S_{4}-S_{3}\right)\left(\nabla_{l} S_{3}\right)\left(p_{m}\right)=0
$$

Hence,

$$
\tilde{S}_{3}=\tilde{S}_{4} \quad \text { or } \quad \lim _{m \rightarrow \infty}\left(\nabla_{l} S_{4}\right)\left(p_{m}\right)=\lim _{m \rightarrow \infty}\left(\nabla_{l} S_{3}\right)\left(p_{m}\right)=0 .
$$

(1). In the case where $\tilde{S}_{3}=\tilde{S}_{4}$. From Theorem 3.1, we have

$$
0=\lim _{m \rightarrow \infty}\left\{\sum_{i, j, k, \alpha}\left(h_{i j k}^{\alpha}\right)^{2}+(S+2 c) S+2 S_{3} S_{4}\right\}\left(p_{m}\right)=\sup \sum_{i, j, k, \alpha}\left(h_{i j k}^{\alpha}\right)^{2}+\left(\frac{3}{2} S+2 c\right) S .
$$

Hence,

$$
\sup \sum_{i, j, k, \alpha}\left(h_{i j k}^{\alpha}\right)^{2}=-\left(\frac{3}{2} S+2 c\right) S
$$


From Theorem 3.1, we have

$$
\begin{aligned}
\sum_{i, j, k, \alpha}\left(h_{i j k}^{\alpha}\right)^{2} & =-(S+2 c) S-2 S_{3} S_{4} \\
& =-\left(\frac{3}{2} S+2 c\right) S+\frac{1}{2}\left(S_{3}-S_{4}\right)^{2} \\
& \geq-\left(\frac{3}{2} S+2 c\right) S .
\end{aligned}
$$

Hence, we have

$$
\inf \sum_{i, j, k, \alpha}\left(h_{i j k}^{\alpha}\right)^{2} \geq-\left(\frac{3}{2} S+2 c\right) S=\sup \sum_{i, j, k, \alpha}\left(h_{i j k}^{\alpha}\right)^{2},
$$

that is,

$$
\sum_{i, j, k, \alpha}\left(h_{i j k}^{\alpha}\right)^{2} \equiv-\left(\frac{3}{2} S+2 c\right) S
$$

is constant. Therefore, $S_{3} \equiv S_{4}$ on $M^{2}$ and they are constant. Hence, on $M^{2}$,

$$
\nabla_{l} S_{3}=\nabla_{l} S_{4}=0
$$

From (3.25), we have $\sum_{i, j, k, \alpha}\left(h_{i j k}^{\alpha}\right)^{2}=0$ on $M^{2}$. According to (3.36), we have $S=\frac{-4 c}{3}$.

(2). In the case where $\tilde{S}_{3} \neq \tilde{S}_{4}$. We have, for $l=1,2$,

$$
\lim _{m \rightarrow \infty} \nabla_{l} S_{3}\left(p_{m}\right)=\lim _{m \rightarrow \infty} \nabla_{l} S_{4}\left(p_{m}\right)=0 .
$$

From $\left|\nabla S_{\alpha}\right|^{2}=4 S_{\alpha} \sum_{i, j, k}\left(h_{i j k}^{\alpha}\right)^{2}$, we have

$$
\lim _{m \rightarrow \infty} S_{3} \sum_{i, j, k}\left(h_{i j k}^{3}\right)^{2}\left(p_{m}\right)=\lim _{m \rightarrow \infty} S_{4} \sum_{i, j, k}\left(h_{i j k}^{4}\right)^{2}\left(p_{m}\right)=0,
$$

that is

$$
\tilde{S}_{3} \lim _{m \rightarrow \infty} \sum_{i, j, k}\left(h_{i j k}^{3}\right)^{2}\left(p_{m}\right)=\tilde{S}_{4} \lim _{m \rightarrow \infty} \sum_{i, j, k}\left(h_{i j k}^{4}\right)^{2}\left(p_{m}\right)=0 .
$$

If

$$
\lim _{m \rightarrow \infty} \sum_{i, j, k}\left(h_{i j k}^{3}\right)^{2}\left(p_{m}\right)=\lim _{m \rightarrow \infty} \sum_{i, j, k}\left(h_{i j k}^{4}\right)^{2}\left(p_{m}\right)=0,
$$

we have

$$
\sup \sum_{i, j, k, \alpha}\left(h_{i j k}^{\alpha}\right)^{2}=0
$$

Hence, from Theorem 3.1, we have 


$$
0=S(S+2 c)+2 S_{3} S_{4}=S\left(\frac{3}{2} S+2 c\right)-\frac{1}{2}\left(S_{3}-S_{4}\right)^{2} .
$$

Hence $S>\frac{-4 c}{3}$ and $S_{3} S_{4}$ is constant. Hence $S_{3}$ and $S_{4}$ are constant because $S=S_{3}+S_{4}$ and $S_{3} S_{4}$ are constant. Since $S>0$, we can assume $S_{3}>0$. From the proof of Theorem 3.1, we have, for $\alpha=3,4$,

$$
0=\frac{1}{2} \Delta S_{\alpha}=\sum_{i, j, k}\left(h_{i j k}^{\alpha}\right)^{2}+(S+2 c) S_{\alpha}+S_{3} S_{4} .
$$

Hence,

$$
(S+2 c) S_{\alpha}+S_{3} S_{4}=0
$$

Therefore, $S=-2 c$ and $M^{2}$ is the hyperbolic cylinder of the totally geodesic hypersurface $\mathbf{H}_{1}^{3}(c)$ from the Theorem due to Ishihara [8].

Next we can assume $\lim _{m \rightarrow \infty} \sum_{i, j, k}\left(h_{i j k}^{4}\right)^{2}\left(p_{m}\right) \neq 0$ without loss of the generality. We have $\tilde{S}_{4}=0$. Because $S=S_{3}+S_{4}>0$ is constant, we have $\tilde{S}_{3} \neq 0$. Hence,

$$
\lim _{m \rightarrow \infty} \sum_{i, j, k}\left(h_{i j k}^{3}\right)^{2}\left(p_{m}\right)=0 .
$$

We shall prove $S=\frac{-10 c}{11}$ in this case. Since $\tilde{S}_{4}=0$, we have

$$
\lim _{m \rightarrow \infty} \sum_{i, j, k, \alpha}\left(h_{i j k}^{\alpha}\right)^{2}\left(p_{m}\right)+(2 c+S) S=0 .
$$

Hence,

$$
\sup \sum_{i, j, k, \alpha}\left(h_{i j k}^{\alpha}\right)^{2}=-(S+2 c) S
$$

From (3.31) and (3.32), we know that $\lim _{m \rightarrow \infty}\left|\nabla \sum_{i, j, k, \alpha}\left(h_{i j k}^{\alpha}\right)^{2}\right|=0$. Hence,

$$
\lim _{m \rightarrow \infty} \sum_{i, j, k} h_{i j k}^{4} h_{i j k l}^{4}\left(p_{m}\right)=0 \quad \text { for } \quad l=1,2,
$$

because of $\lim _{m \rightarrow \infty} \sum_{i, j, k}\left(h_{i j k}^{3}\right)^{2}\left(p_{m}\right)=0$. Thus we conclude

$$
\tilde{h}_{111}^{4} \tilde{h}_{2211}^{4}=-\tilde{h}_{222}^{4} \tilde{h}_{1121}^{4}, \quad \tilde{h}_{111}^{4} \tilde{h}_{1112}^{4}=\tilde{h}_{222}^{4} \tilde{h}_{1122}^{4} .
$$

According to Lemma 2 we have

$$
\tilde{h}_{1122}^{4}=\tilde{h}_{2211}^{4}, \quad \tilde{h}_{1112}^{4}=\tilde{h}_{1121}^{4} .
$$

Hence

$$
\left(\tilde{h}_{111}^{4}\right)^{2} \tilde{h}_{2211}^{4}+\left(\tilde{h}_{222}^{4}\right)^{2} \tilde{h}_{2211}^{4}=0 .
$$


Since $\sum_{i, j, k}\left(\tilde{h}_{i j k}^{4}\right)^{2} \neq 0$, then $\tilde{h}_{2211}^{4}=\tilde{h}_{1122}^{4}=\tilde{h}_{1121}^{4}=\tilde{h}_{1112}^{4}=0$. On the other hand, since $S$ is constant we have

$$
\sum_{i, j, \alpha} h_{i j}^{\alpha} h_{i j l k}^{\alpha}+\sum_{i, j, \alpha} h_{i j k}^{\alpha} h_{i j l}^{\alpha}=0 \quad \text { for any } \quad l, k .
$$

Hence

$$
\begin{aligned}
2 \tilde{\lambda} \tilde{h}_{1112}^{3} & =0, \\
2 \tilde{\lambda} \tilde{h}_{1121}^{3} & =0, \\
2 \tilde{\lambda} \tilde{h}_{1122}^{3} & =-\frac{1}{2} \sum_{i, j, k, \alpha}\left(\tilde{h}_{i j k}^{\alpha}\right)^{2}, \\
2 \tilde{\lambda} \tilde{h}_{2211}^{3} & =\frac{1}{2} \sum_{i, j, k, \alpha}\left(\tilde{h}_{i j k}^{\alpha}\right)^{2} .
\end{aligned}
$$

We infer

$$
\left(\tilde{h}_{1122}^{3}+\tilde{h}_{2211}^{3}\right)^{2}+\left(\tilde{h}_{1112}^{3}+\tilde{h}_{1121}^{3}\right)^{2}=0 .
$$

Therefore, from Lemma 2 we have

$$
\sum_{i, j, k, l, \alpha}\left(\tilde{h}_{i j k l}^{\alpha}\right)^{2}=(S+2 c)^{2} S .
$$

From Theorem 3.1, we have

$$
\begin{aligned}
& \frac{1}{2} \Delta \sum_{i, j, k, \alpha}\left(h_{i j k}^{\alpha}\right)^{2} \\
& =-\Delta\left(S_{3} S_{4}\right) \\
& =-S_{3} \Delta S_{4}-2 \nabla S_{3} \cdot \nabla S_{4}-S_{4} \Delta S_{3} .
\end{aligned}
$$

Hence

$$
\lim _{m \rightarrow \infty} \frac{1}{2} \Delta \sum_{i, j, k, \alpha}\left(h_{i j k}^{\alpha}\right)^{2}\left(p_{m}\right)=-\lim _{m \rightarrow \infty}\left(S_{3} \Delta S_{4}\right)\left(p_{m}\right)=2 S(S+2 c) S
$$

because of $\tilde{S}_{4}=0$ and $\lim _{m \rightarrow \infty} \sum_{i, j, k}\left(h_{i j k}^{3}\right)^{2}\left(p_{m}\right)=0$. From (3.37), (3.38), (3.39) and Theorem 3.2, we have

$$
2(S+2 c) S^{2}=\frac{1}{2} \lim _{m \rightarrow \infty} \Delta \sum_{i, j, k, \alpha}\left(h_{i j k}^{\alpha}\right)^{2}\left(p_{m}\right)=(S+2 c)^{2} S-\left(\frac{9}{2} S+7 c\right)(S+2 c) S .
$$

Thus $S=-\frac{10 c}{11}$. From the above proof, we know that $S=\frac{-4 c}{3}$ if and only if $S_{3}=S_{4}$ is constant on $M^{2}$ and $\sum_{i, j, k, \alpha}\left(h_{i j k}^{\alpha}\right)^{2} \equiv 0$. By making use of the similar method to one which was used in [7] by Chern, do Carmo and Kobayashi, we can prove that $M^{2}$ is isometric to the hyperbolic Veronese surface. We complete the proof of Main Theorem. 


\section{REFERENCES}

1. E. Calabi, Examples of Bernstein problems for nonlinear equations, Proc. Symp. Pure and Appl. Math. 15 (1970), 223-230.

2. Q. M. Cheng, Complete spacelike submanifolds in a de Sitter space with parallel mean curvature vector, Math. Z. 206 (1991), 333-339.

3. Q. M. Cheng, Complete maximal spacelike hypersurfaces of $\mathbf{H}_{1}^{4}(c)$, Manuscripta Math. 82 (1994), 149-160.

4. Q. M. Cheng, Spacelike surfaces in an anti-de Sitter space, Colloquium Math. 46 (1994), 201-208.

5. Q. M. Cheng and H. Nakagawa, Totally umbilical hypersurfaces, Hiroshima Math. J. 20 (1990), 1-10.

6. S. Y. Cheng and S. T. Yau, Maximal spacelike hypersurfaces in the Lorentz-Minkowski space, Ann. of Math. 104 (1976), 407-419.

7. S. S. Chern, M. do Carmo and S. Kobayashi, Minimal submanifolds of a sphere with second fundamental form of constant length, in Functional analysis and related fields (Springer-Verlag, 1970) 59-75.

8. T. Ishihara, Maximal spacelike submanifolds of a pseudoriemannian space form of constant curvature, Michigan Math. J. 35 (1988), 345-352.

9. H. Omori, Isometric immersion of Riemannian manifolds, J. Math. Soc. Japan 19 (1967), 205-214.

10. A. E. Treibergs, Entire hypersurfaces of constant mean curvature in Minkowski 3-space, Invent. Math. 66 (1982), 39-56.

11. J. Simons, Minimal varieties in Riemannian manifolds, Ann. of Math. 88 (1968), 62-105.

12. S. T. Yau, Harmonic functions on complete Riemannian manifolds, Comm. Pure and Appl. Math. 28 (1975), 201-228. 